\title{
AN UNUSUAL CASE OF PORTAL, SPLENIC AND MESENTERIC VENOUS THROMBOSIS PRESENTING WITH ACUTE ABDOMEN
}

\author{
Yeoh $\mathrm{CN}^{1}, \mathrm{R}$ Nadiah${ }^{2}$, Cheah $S K^{1}$, Wan Mat WR ${ }^{1}, M$ Maaya $^{1}, A R$ Raha $^{1}$ \\ ${ }^{1}$ Department of Anaesthesiology and Intensive Care, Universiti Kebangsaan Malaysia Medical Centre, Kuala Lumpur, \\ Malaysia \\ ${ }^{2}$ Department of Surgery, Universiti Kebangsaan Malaysia Medical Centre, Kuala Lumpur, Malaysia
}

\section{Correspondence:}

Chih Nie Yeoh

Department of Anaesthesiology and Intensive Care,

Universiti Kebangsaan Malaysia Medical Centre (UKMMC),

Jalan Yaacob Latif, Bandar Tun Razak,

56000 Cheras, Kuala Lumpur, Malaysia

Tel: +60391455783

Fax: +60391456585

Email:yeoh@ukm.edu.my

\begin{abstract}
Porto-spleno-mesenteric vein thrombosis is a rare, life-threatening condition of extrahepatic portal venous system thrombosis. We report a rare case of a 49 -year-old lady with late presentation of acute portal vein thrombosis in a non-cirrhotic liver with an incidental finding of left adnexal teratoma. She presented with a one-week history of severe abdominal pain associated with vomiting and diarrhea. She gave no history of prior risk for venous thromboembolism or liver diseases. Physical examination revealed a tender mass extending from suprapubic to left iliac fossa. Abdominal computed tomography scans showed a well-defined fat-containing left adnexal mass, likely a benign teratoma, with no involvement of surrounding structures or calcification. There was evidence of porto-splenic-mesenteric vein thrombosis with liver infarction, bowel and splenic ischemia. Management of the extensive thrombosis causing multi-organ failure includes resuscitation, supportive care and treatment of thrombosis. Treatment options include early anticoagulation and if feasible, thrombolysis.
\end{abstract}

Keywords: Acute Abdomen, Mesenteric Vein Thrombosis, Non-Cirrhotic Vein Thrombosis, Portal Vein Thrombosis, Splenic Vein Thrombosis

\section{Introduction}

Portal vein thrombosis (PVT) is a rare condition that is potentially life-threatening. Its clinical manifestation can extend to the intra-hepatic portal veins or to splenic or mesenteric veins leading to intestinal infarction (1). Hematological hyper-coagulable states account for $40-60 \%$ of non-cirrhotic portal vein thrombosis (1).

\section{Case Report}

A 49-year-old lady presented to our institution with oneweek history of abdominal pain that was associated with multiple bouts of diarrhea and vomiting. The pain was described as colicky, originating from the epigastrium and radiating to the lower abdomen. She has hypertension and diabetes mellitus. She did not give a history of previous venous thromboembolic events. There was no family history of malignancy and she did not consume any other medications apart from her anti-hypertensive and oral hypoglycemic agents. There was no history of taking traditional medications and she was not on any contraception. She does not smoke or take alcohol.

Upon presentation, she was afebrile, tachycardic and normotensive. Abdominal palpation revealed a tender mass at suprapubic region extending to the left iliac fossa. She did not exhibit any features of chronic liver disease. Splenomegaly or hepatomegaly was not present. Other systemic examinations were unremarkable.

Full blood counts showed elevated white cell count $(27 \mathrm{x}$ $10^{\wedge} 9 / \mathrm{L}$ ) with predominant neutrophilia (89.8\%) suggestive of possible bacterial infection, thrombocytosis of 658 $x 10^{\wedge} 9$ /L with a normal hemoglobin (14 g/dL) but low mean corpuscular hemoglobin $(19.8 \mathrm{pg})$ and low mean cell volume (65 fl). Her peripheral blood smear showed normal hemoglobin with hypochromic microcytic red cells, 
moderate to severe anisopoikilocytosis and ecchinocytes, presence of occasional pencil cells and target cells, left shift of myelocytes and metamyelocytes, anisocytosis and large giant platelets. These features were reported to be suggestive of underlying thalassemia trait or hemoglobinopathy. Serum procalcitonin was elevated $(6.03 \mathrm{ng} / \mathrm{ml})$ suggestive of severe systemic inflammatory response or sepsis. She also had acute kidney injury with hyperkalemia $(6.5 \mathrm{mmol} / \mathrm{L})$, raised urea $(12.3 \mathrm{mmol} / \mathrm{L})$ and raised serum creatinine $(143 \mu \mathrm{mol} / \mathrm{L})$.

Liver function test showed elevated total protein level (85 g/L) with low normal albumin (34 g/L). Alkaline phosphatase level was mildly elevated at $177 \mathrm{U} / \mathrm{L}$ with normal total bilirubin level $(13.5 \mu \mathrm{mol} / \mathrm{L})$ and normal alanine aminotransferase level $(28 \mathrm{U} / \mathrm{L})$.

Coagulation screen revealed a raised international normalized ratio (INR) of 1.5 , raised fibrinogen level $(4.9 \mathrm{~g} / \mathrm{L})$ and D-dimer (>20 $\mu \mathrm{g} / \mathrm{ml})$. Elevated D-dimer is suggestive of ongoing thrombus formation and breakdown with raised fibrin degradation products. Arterial blood gas showed metabolic acidosis and a progressively increasing lactate level from $1.7 \mathrm{mmol} / \mathrm{L}$ to $14 \mathrm{mmol} / \mathrm{L}$. This prompted the suspicion of bowel ischemia. Serum amylase and $\mathrm{HbA1C}$ were otherwise normal.

Computed tomography (CT) scan of the abdomen revealed extensive superior mesenteric vein, splenic and portal vein thrombosis with short segment thrombosis within the aorta at the level of the third lumbar vertebrae along with small bowel wall thickening with mesenteric fat streakiness and mesenteric vessels engorgement indicative of small bowel ischemia. Hypodensities in segment VI and VII of liver were seen with heterogenous enhancement of spleen, likely showing liver and splenic ischemia/infarct (Figure $1 \mathrm{a}$ and $1 \mathrm{~b}$ ). There was a well-defined thin-walled predominantly fat-containing lesion seen at the left adnexa, measuring $5.7 \mathrm{~cm} \times 5.8 \mathrm{~cm} \mathrm{X} 6.0 \mathrm{~cm}$ and without calcification, reported to be likely a teratoma. It was in close proximity with the rectum and small bowel with no evidence of involvement. The right ovary was normal but the left ovary was not seen.

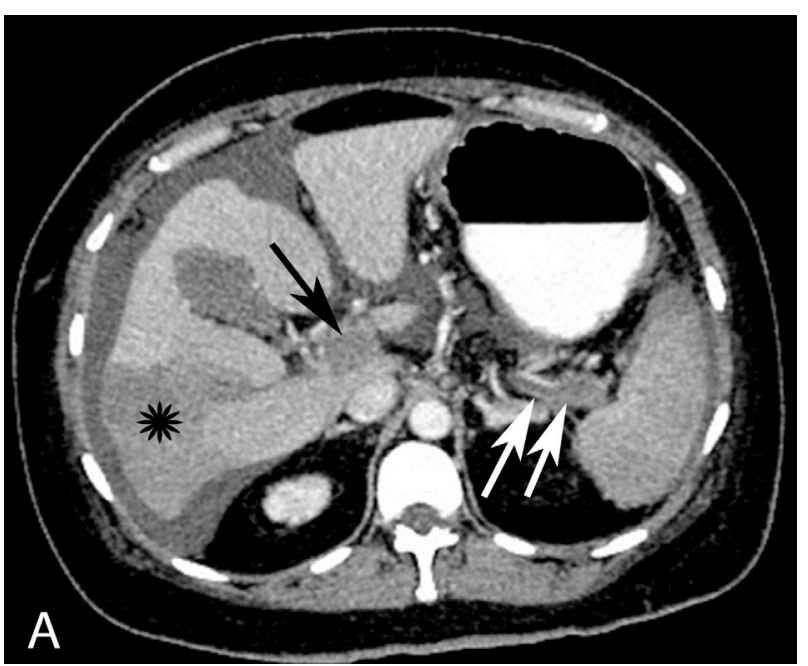

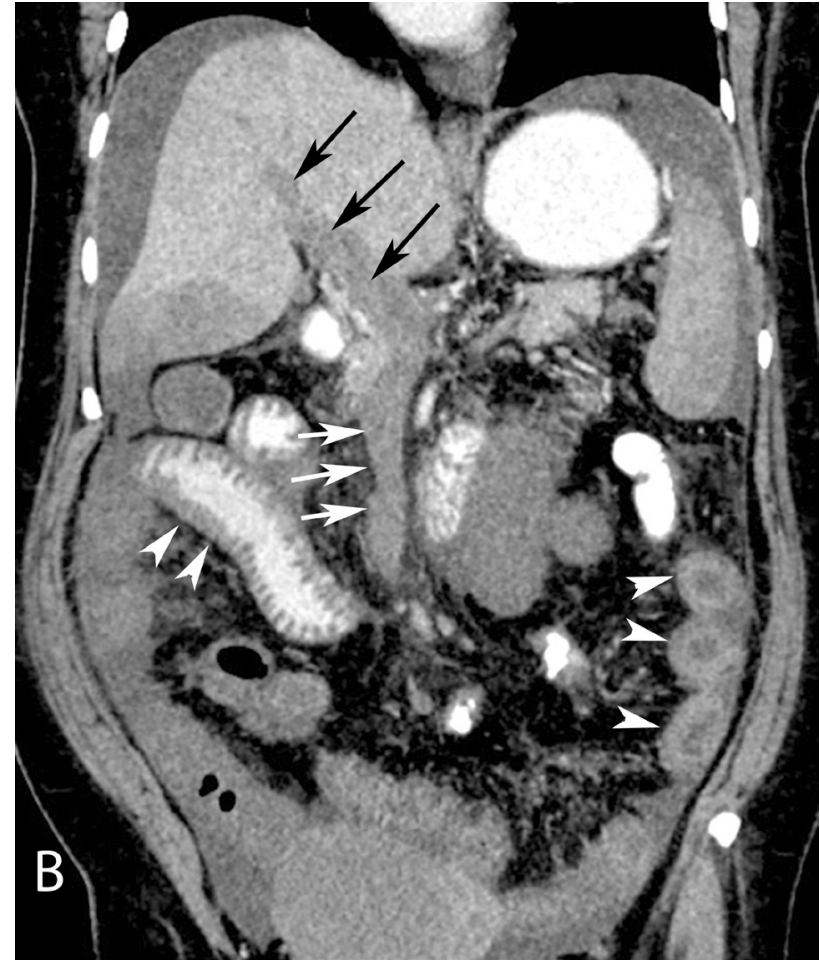

Figure 1: Contrast enhanced CT abdomen of the patient in axial (a) and coronal (b). (a) There is filling defect in the proximal splenic vein (white arrows) as well as in the portal confluence (black arrow). There is geographical heterogeneity of the liver parenchyma (asterisk) indicating liver parenchymal infarct. Ascites is also seen at the perihepatic region. (b) Long segment filling defect is also seen throughout the right portal vein and main portal vein (black arrows) and superior mesenteric vein (white arrows). The small bowel walls are edematous most likely due to venous congestion/infarct (white arrowheads).

In view of worsening metabolic acidosis and renal function, she was mechanically ventilated and renal replacement therapy initiated in the intensive care unit. Intravenous (IV) heparin infusion was commenced for the extensive thrombosis. She was given antibiotics for possible sepsis from the ischemic bowels. Despite active treatment, her hemodynamics remained unstable requiring triple inotropic support and persistent hyperkalemia with severe metabolic acidosis. She was unable to proceed for a planned exploratory laparotomy. Her condition deteriorated rapidly and she succumbed within 48 hours of presentation to our center.

\section{Discussion}

Portal vein thrombosis (PVT) was first reported in 1868 (1). The lifetime risk of getting PVT in the general population is documented to be about $1 \%(2)$. However, accurate epidemiological data on PVT is not readily available.

Portal vein thrombosis may be asymptomatic or present with nonspecific symptoms such as abdominal pain, or lifethreatening manifestations like gastrointestinal bleeding 
or hemorrhagic intestinal infarction (3). Thrombosis of the spleno-mesenteric-portal axis is classified into four categories by Jamieson: (i) Partial or complete thrombosis limited to the portal vein beyond the confluence of the splenic and superior mesenteric vein, (ii) Extension of thrombus into the superior mesenteric vein, but with patent mesenteric vessels, (iii) Diffuse thrombosis of splanchnic venous system, but with large-caliber collaterals, and (iv) Extensive splanchnic venous thrombosis, but with small collaterals (3).

Portal vein thrombosis may be acute or chronic. In acute PVT, patients usually present with abdominal pain indicating possible extension of thrombus into the mesenteric vein (2). When thrombosis extends to mesenteric venous arches, it can cause intestinal infarction. Once intestinal infarction occurs, the mortality rate is at least $50 \%$ (3). Intestinal infarction depends on the extent of superior mesenteric vein thrombosis and the collateral circulation provided by the venous arcades. If the mesenteric venous flows are occluded, the arterioles develop reflex vasoconstriction which can lead to persistence of ischemia and lastly, intestinal infarction (3). Acute PVT is more commonly associated with sepsis compared to chronic PVT. Nearly $40 \%$ of patients with acute PVT were reported to have concurrent systemic or intra-abdominal sepsis (4). However, chronic PVT is only evident clinically following signs and symptoms of portal hypertension $(1,4)$.

Risk factors of PVT can be classified as local or systemic. Up to $80 \%$ of idiopathic PVT has an identifiable systemic cause. In these patients, $60 \%$ were found to have general thrombophilic disorders and $40 \%$ were due to local causes (4). In the event of unknown etiology, investigations of a systemic cause must be done, which includes excluding malignancies such as myeloproliferative disorder and other prothrombotic tendencies (Factor V Leiden deficiency, antiphospholipid syndrome, deficiencies of Protein C, Protein S and antithrombin III, G20210A prothrombin gene mutation and hyperhomocysteinemia). We could not exclude the possibility of hemoglobinopathies given the limited time available with the patient before she deteriorated. Tests such as peripheral blood smear or hemoglobin electrophoresis take time to process.

Chronic myeloproliferative neoplasms (MPN) have been described as the commonest systemic cause of splanchnic vein thrombosis (4). In the majority of patients with obvious MPN and in $40 \%$ of patients without obvious MPN, the somatic mutation JAK2 V617F is detected $(5,6)$. $70 \%$ of cirrhotic patients with PVT show genetic thrombophilic predisposition, with higher incidence of the G20210A prothrombin gene mutation (4).

Complaints of suprapubic pain with a palpable mass led to the finding of a left adnexal mass, likely a teratoma. We are unsure if the mass contributed to the PVT or if its presence was a coincidence. It was reported to be in close proximity with adjacent rectum and small bowel with no evidence of involvement. Neither was it part of the uterus. We postulated it was part of the left ovary. Ovarian teratomas are the most common germ cell neoplasm and based on ultrasonography or CT scan, her left ovary was not visualized. The teratoma itself in $\mathrm{CT}$ was reported to be well-defined, fat-filled, with no calcification and no involvement of surrounding structures, which is suggestive of its benign nature and is also diagnostic of mature cystic teratoma (dermoid cyst)(7).

Mature cystic teratoma can develop complications such as rupture, torsion or malignant transformation. Ovarian torsion is the commonest complication and may cause pelvic pain in female patients. The ovarian pedicle is twisted causing arterial, venous and lymphatic obstruction resulting in circulatory stasis. CT findings show a twisted ovarian pedicle, thickened Fallopian tube, enlarged affected ovary, cystic wall thickening, ascites, enlarged veins and uterine deviation to the twisted side (8). Malignant transformation is rare (1-2\%) and CT findings include features of irregularly marginated soft tissue component extending into or within the tumor wall (8). Rupture of ovarian teratoma causes spillage of liquefied sebaceous contents into the peritoneum causing irritation and inflammation. These would appear as ascites, flattened shape of the teratoma with surrounding inflammatory masses involving the omentum and bowel (8). None of our patient's CT findings suggest any of the above complications.

There are studies showing increased risk for venous thromboembolism with ovarian tumor (9). Most mature cystic teratomas are asymptomatic and grow very slowly (7). Although benign, we postulate that this slow-growing mass in the pelvis increased the risk of thrombosis in two ways: one, via the compressive effect to the surrounding vasculature causing splanchnic circulatory stasis, and two, possible expression of tissue factor (TF) by mature cystic teratoma. TF as a transmembrane receptor and primary initiator of coagulation can cause a hypercoagulable state when activated (10).

Non-invasive imaging techniques such as Doppler ultrasound, computed tomography (CT) and magnetic resonance imaging (MRI) are tools to diagnose PVT and determine its cause. Improvements in imaging techniques have allowed PVT to be diagnosed more frequently (3). CT scan may be more useful than ultrasound in demonstrating porto-systemic collaterals and the development of a cavernoma, both suggestive of well-established PVT (4). CT abdomen with portal venous phase contrast is highly sensitive and specific. It is reliable in diagnosing mesenteric vein thrombosis and is less invasive compared to the gold standard of splanchnic angiography. However, splanchnic angiography is still useful because it allows for more accurate differentiation of arterial from venous forms of acute mesenteric infarction besides providing access for intra-arterial vasodilator therapy (11).

Magnetic resonance angiography (MRA) may also assist in confirming PVT. Magnetic resonance (MR) portography is superior to color Doppler ultrasound in detecting partial occlusion and thrombosis of the main portal venous 
vessels. It is also better than color Doppler in identifying porto-splenic collaterals and portal venous vessels (2).

Apart from identifying the underlying cause for PVT, management of PVT is to reverse the thrombosis, prevent worsening of thrombosis and manage its complications, such as gastrointestinal or biliary-related complications (4). Options of treatment include anticoagulation and when indicated, thrombolysis (1).

Patients with acute PVT treated with thrombolytic therapy have been reported to have resolution of thrombosis (4). Thrombolysis can be given into the systemic venous circulation, the superior mesenteric artery or into the portal vein via a transjugular or transhepatic route. Thrombolysis has been shown to be effective when initial heparin therapy has failed (4).

Vascular recanalization is rare without any treatment. Vascular patency has been restored in $80 \%$ of patients who were given anticoagulant therapy. Treatment with anticoagulant should continue for a minimum of 6 months in patients with no thrombotic risks and for life in patients who have the risk factors (3). Plessier et al in the year 2010 found that patients with acute PVT who were treated early with anticoagulant have a high rate of recanalization and no thrombus extension. Risk of severe bleeding was not increased while incidence of intestinal infarction was only $3 \%$ when anticoagulation was initiated early. Also, early anticoagulation is effective in preventing extension of PVT but is less effective in inducing patency in patients with complete extra-hepatic portal vein occlusion. Therefore, for patients who have no resolution in thrombosis of the portal vein despite being on anticoagulants, portal hypertension may develop permanently. However, for patients with splenic vein thrombosis and ascites on presentation, recanalization from anticoagulants is unlikely. Therefore other treatment options should be considered (12).

Our patient had received intravenous heparin as part of the management of the PVT. We did not give thrombolysis after discussion with the primary physician and surgical team because the thrombosis was too extensive, there was multi-organ failure and the patient was in sepsis. Instead, we started the patient on intravenous heparin. Administering thrombolysis when the patient was in sepsis and multi-organ failure was deemed risky. Risk of catastrophic bleeding and disseminated intravascular coagulopathy were our concerns. Despite the scarcity of studies conducted on anticoagulant therapy in acute PVT, there are small studies stating the benefit of prescribing anticoagulant in achieving recanalization of the vessels as well as in preventing long-term complications (3).

Limitations while managing this case included difficulty with consent to a postmortem by the next of kin. Hence, we were not able to obtain further information. We recommend performing tests for tumor markers such as carcioembrionic antigen (CEA), alpha-fetoprotein, beta human chorionic gonadotropin (B hCG) and cancer antigen
125 (Ca 125) to improve detection of malignancy and its origin. Unfortunately these results were not available to us.

\section{Conclusion}

PVT occurs more frequently in patients with cirrhosis or underlying malignancy. On its own, the presentation is nonspecific and can mimic other more common causes of acute abdomen. Therefore a high index of suspicion is needed to diagnose PVT. Management of PVT ultimately depends on time of presentation and diagnosis. If the patient presents late or is diagnosed late, multi-organ failure may already occur and the patient could become gravely ill. Mortality and morbidity at this point could be high. We recommend that the emphasis of treatment be on resuscitation, supportive care and treating the PVT. Diagnosing the cause of thrombosis should run concurrent with treatment but never supersede active resuscitation, supportive care and treating the PVT. Whenever possible, thrombolysis should be considered but risks of giving thrombolysis needs to be weighed against its benefit, and also, versus the option of giving anticoagulant.

\section{Acknowledgements}

The authors would like to thank Associate Professor Dr. Faizah Mohd Zaki, esteemed clinical radiologist and lecturer of PPUKM, who had helped us improve this paper by providing guidance on the radiological images.

\section{Competing interests}

The authors declare that they have no competing interests.

\section{Financial support}

The authors declare that they have no financial support

\section{Informed Consent}

Written informed consent was obtained from the patient's next of kin for inclusion in this report. Research and ethics committee approval for case reports is not a requirement in Universiti Kebangsaan Malaysia Medical Centre (UKMMC).

\section{References}

1. Grouzi E, Politou M, Douramani P, Merkouri E, Gialeraki A, Brountzos $\mathrm{H}$, et al. Portal, splenic and mesenteric vein thrombosis in a patient double heterozygous for factor $\mathrm{V}$ Leiden and prothrombin G20210A mutation. Blood Coagul Fibrinolysis. Dec 2009; 20(8):722-5.

2. Chawla Y, Duseja A and Dhiman RK. Review article: the modern management of portal vein thrombosis. Aliment Pharmacol Ther. Nov 2009; 30(9): 881-894.

3. Sacerdoti D, Serianni G, Gaiani S, Bolognesi M, Bombonato G, and Gatta A. Thrombosis of the portal venous system. J Ultrasound. Mar 2007; 10(1): 12-21.

4. Webster GJM, Burroughs AK, Riordan SM. Review article: portal vein thrombosis - new insights into 
aetiology and management. Aliment Pharmacol Ther. Jan 2005; 21(1):1-9.

5. De Stefano V, Za T, Ciminello A, Betti S, and Rossi E. Causes of adult splanchnic vein thrombosis in the mediterranean area. Mediterr J Hematol Infect Dis. Dec 2011; 3(1): e2011063.

6. Primignani M, Barosi G, Bergamaschi G, Gianelli $U$, Fabris F, Reati R, et al. Role of the JAK2 mutation in the diagnosis of chronic myeloproliferative disorders in splanchnic vein thrombosis. Hepatology. Dec 2006; 44(6):1528-1534.

7. Eric KO, Evan SS, Jennifer LH. Ovarian teratomas: Tumour types and imaging characteristics. RadioGraphics. 2001; 21:475-490.

8. Park SB, Kim JK, Kim KR, Cho KS. RadioGraphics. 2008; 28:969-983.

9. Fennerty A. Review: Venous thromboembolic disease and cancer. Postgrad Med J. 2006; 82:642-648.

10. Vidhya R, Lakshminarasimhan S, Madurai PK. Internal jugular venous thrombosis presenting as paraneoplastic syndrome in benign cystic teratoma of ovary: a case report. Int J Reprod Contracept Obstet Gynecol. Jan 2007; 6(1):287-291.

11. Ian GH, Lawarence JB. Mesenteric venous thrombosis. Vasc Med. 2010; 15(5):407-418.

12. Plessier A, Darwish-Murad S, Hernandez-Guerra M, Consigny $\mathrm{Y}$, Fabris F, Trebicka J, et al. Acute portal vein thrombosis unrelated to cirrhosis: A prospective multi-center follow-up study. Hepatology. Jan 2010; 51(1): 210-218. 